\title{
A Probe of Primordial Gravity Waves and Vorticity
}

\author{
Marc Kamionkowski* \\ Department of Physics, Columbia University, 538 West 120th Street, New York, New York 10027
}

Arthur Kosowsky ${ }^{\dagger}$

Harvard-Smithsonian Center for Astrophysics, 60 Garden Street, Cambridge, Massachusetts 02138

and Department of Physics, Lyman Laboratory, Harvard University, Cambridge, Massachusetts 02138

\begin{abstract}
Albert Stebbins
NASA/Fermilab Astrophysics Center, Fermi National Accelerator Laboratory, Batavia, Illinois 60510-0500
\end{abstract}

(Received 19 September 1996)

\begin{abstract}
A formalism for describing an all-sky map of the polarization of the cosmic microwave background is presented. The polarization pattern on the sky can be decomposed into two geometrically distinct components. One of these components is not coupled to density inhomogeneities. A nonzero amplitude for this component of polarization can only be caused by tensor or vector metric perturbations. This allows unambiguous identification of long-wavelength gravity waves or large-scale vortical flows at the time of last scattering. [S0031-9007(97)02705-1]

PACS numbers: 98.70.Vc, 04.30.Nk, 98.80.Cq
\end{abstract}

With the COBE detection of large-angle anisotropy in the cosmic microwave background (CMB), results from numerous balloon-borne and ground-based experiments, and the advent of a new generation of satellite missions, the CMB is becoming an increasingly precise probe of the early Universe. CMB anisotropies will help determine whether density perturbations (scalar modes) are the result of inflation, topological defects, or perhaps some other mechanism. Detection of a stochastic gravitywave background (tensor modes) [1] or vortical motions in the primeval fluid (vector modes) would help to discriminate between these models. Inflation damps out vector modes but will produce some tensor modes and also predicts a specific relationship between the spectrum of the scalar and tensor fluctuations [2]. In contrast, topological defects will produce a mixture of scalar, vector, and tensor modes. Scalar modes give rise to both the observed large-scale structure and CMB fluctuations, while in the foreseeable future we can only expect to observe the consequences of tensor or vector modes through their effects on the CMB. Without a model of primordial fluctuations, the contribution of scalar, vector, and tensor modes to the CMB temperature anisotropy are indistinguishable.

However, any mechanism which produces temperature anisotropies will invariably lead to nonzero polarization as well [3-6]. As we demonstrate in this Letter, this polarization signal can be used to discriminate between scalar and vector or tensor metric perturbations. COBE has already mapped the polarization pattern with an angular resolution of $7^{\circ}$ (although the data have not been analyzed), and MAP [7] will measure the polarization with a resolution of around $0.3^{\circ}$.

In prior work, the autocorrelation and cross correlations between the Stokes parameters $Q$ and $U$ and the tempera- ture $T$ have been considered. Here $Q$ and $U$ are defined with respect to particular orthogonal axes on the celestial sphere. While this formalism does provide a complete description of the polarization, there is no rotationally invariant way to lay down orthogonal basis vectors on a sphere, so the meaning of $Q Q, Q U, U U, Q T$, or $U T$ will depend on absolute positions of the points being correlated rather than just the relative position. Calculations of this sort have been done with a small-angle approximation, since rotational noninvariance disappears when considering only a small patch of the sky. However, this formalism is not optimal for describing the complete temperature and polarization correlations present in full-sky maps.

Here we present a rotationally covariant formalism for describing the polarization pattern on a full sky. The Stokes parameters, defined by the $2 \times 2$ correlation matrix of the electric field of incoming photons, can be described as a tensor field on the celestial sphere. The $Q$ and $U$ parameters, describing linear polarization, are just given by the symmetric trace-free (STF) part of this tensor. For example, in spherical polar coordinates $(\theta, \phi)$, where the spherical metric is $g_{a b}=\operatorname{diag}\left(1, \sin ^{2} \theta\right)$, the polarization tensor is

$$
\mathcal{P}_{a b}(\hat{\mathbf{n}})=\frac{1}{2}\left(\begin{array}{ll}
Q(\hat{\mathbf{n}}) & -U(\hat{\mathbf{n}}) \sin \theta \\
-U(\hat{\mathbf{n}}) \sin \theta & -Q(\hat{\mathbf{n}}) \sin ^{2} \theta
\end{array}\right) .
$$

The " $a b$ " are the tensor indices, and we use standard tensor notation throughout. It is natural to decompose the linear-polarization pattern into STF tensor spherical harmonics [8,9], which constitute a complete orthonormal set of rank-2 STF tensors on the sphere. There are two types of harmonic STF tensors, $Y_{(l m) a b}^{\mathrm{G}}$ and $Y_{(l m) a b}^{\mathrm{C}}$, one of each for every one of the usual spherical harmonics $Y_{(\mathrm{Im})}$ with $l \geq 2$. Two sets of tensor harmonics are required as there are two modes of linear polarization, $Q$ and $U$. 
Since Compton scattering can produce no net circular polarization, the $\mathrm{CMB}$ is expected to have $V=0$, and the $V$ Stokes parameter will not be considered further.

The harmonic expansion of an all-sky map of the CMB temperature and polarization can be written

$$
\begin{aligned}
\frac{T(\hat{\mathbf{n}})}{T_{0}} & =1+\sum_{l m} a_{(l m)}^{\mathrm{T}} Y_{(l m)}(\hat{\mathbf{n}}) \\
\frac{\mathcal{P}_{a b}(\hat{\mathbf{n}})}{T_{0}} & =\sum_{l m}\left[a_{(l m)}^{\mathrm{G}} Y_{(l m) a b}^{\mathrm{G}}(\hat{\mathbf{n}})+a_{(l m)}^{\mathrm{C}} Y_{(l m) a b}^{\mathrm{C}}(\hat{\mathbf{n}})\right] .
\end{aligned}
$$

The mode amplitudes are given by

$$
\begin{aligned}
& a_{(l m)}^{\mathrm{T}}=\frac{1}{T_{0}} \int d \hat{\mathbf{n}} T(\hat{\mathbf{n}}) Y_{(l m)}^{*}(\hat{\mathbf{n}}), \\
& a_{(l m)}^{\mathrm{G}}=\frac{1}{T_{0}} \int d \hat{\mathbf{n}} \mathcal{P}_{a b}(\hat{\mathbf{n}}) Y_{(l m)}^{\mathrm{G} a b *}(\hat{\mathbf{n}}), \\
& a_{(l m)}^{\mathrm{C}}=\frac{1}{T_{0}} \int d \hat{\mathbf{n}} \mathcal{P}_{a b}(\hat{\mathbf{n}}) Y_{(l m)}^{\mathrm{C} a b *}(\hat{\mathbf{n}}),
\end{aligned}
$$

which can be derived from the orthonormality properties

$$
\begin{gathered}
\int d \hat{\mathbf{n}} Y_{(l m)}^{*}(\hat{\mathbf{n}}) Y_{\left(l^{\prime} m^{\prime}\right)}(\hat{\mathbf{n}})=\delta_{l l^{\prime}} \delta_{m m^{\prime}}, \\
\int d \hat{\mathbf{n}} Y_{(l m) a b}^{\mathrm{G} *}(\hat{\mathbf{n}}) Y_{\left(l^{\prime} m^{\prime}\right)}^{\mathrm{G} a b}(\hat{\mathbf{n}})=\delta_{l l^{\prime}} \delta_{m m^{\prime}}, \\
\int d \hat{\mathbf{n}} Y_{(l m) a b}^{\mathrm{C} *}(\hat{\mathbf{n}}) Y_{\left(l^{\prime} m^{\prime}\right)}^{\mathrm{C} a b}(\hat{\mathbf{n}})=\delta_{l l^{\prime}} \delta_{m m^{\prime}}, \\
\int d \hat{\mathbf{n}} Y_{(l m) a b}^{\mathrm{G} *}(\hat{\mathbf{n}}) Y_{\left(l^{\prime} m^{\prime}\right)}^{\mathrm{C} a b}(\hat{\mathbf{n}})=0 .
\end{gathered}
$$

Here $T_{0}$ is the cosmological mean CMB temperature, and we are assuming $Q$ and $U$ are measured in brightness temperature units rather than flux units.

The two geometrically distinct tensor harmonics are

$$
\begin{aligned}
& Y_{(l m) a b}^{\mathrm{G}}=N_{l}\left(Y_{(l m): a b}-\frac{1}{2} g_{a b} Y_{(l m): c}{ }^{c}\right), \\
& Y_{(l m) a b}^{\mathrm{C}}=\frac{N_{l}}{2}\left(Y_{(l m): a c} \epsilon_{b}^{c}+Y_{(l m): b c} \epsilon_{a}^{c}\right) .
\end{aligned}
$$

Here $N_{l}=\sqrt{2(l-2) ! /(l+2) !}$ is a normalization factor, $\epsilon_{a b}$ is the completely antisymmetric tensor, and ":" indicates a covariant derivative on the sphere. In two dimensions, any STF tensor can be uniquely decomposed into a part of the form $A_{: a b}-(1 / 2) g_{a b} A_{: c}{ }^{c}$ and another part of the form $B_{: a c} \epsilon_{b}^{c}+B_{: b c} \epsilon_{a}^{c}$ where $A$ and $B$ are two scalar functions. This decomposition is quite similar to the decomposition of a vector field into a part which is the gradient of a scalar field and a part which is the curl of a vector field; hence we use the notation G for "gradient" and C for "curl." Since the $Y_{(l \mathrm{~m})}$ 's provide a complete basis for scalar functions on the sphere, the $Y_{(l m) a b}^{\mathrm{G}}$, s and $Y_{(l m) a b}^{\mathrm{C}}$ 's provide a complete basis for G-type and C-type STF tensors, respectively. This G/C decomposition is also known as the scalar/pseudoscalar decomposition [9].

In $(\theta, \phi)$ coordinates, where Eq. (1) holds, the harmonics are given explicitly by

$$
\begin{aligned}
& Y_{(l m) a b}^{\mathrm{G}}(\hat{\mathbf{n}})=\frac{N_{l}}{2}\left(\begin{array}{ll}
W_{(l m)} & X_{(l m)} \sin \theta \\
X_{(l m)} \sin \theta & -W_{(l m)} \sin ^{2} \theta
\end{array}\right), \\
& Y_{(l m) a b}^{\mathrm{C}}(\hat{\mathbf{n}})=\frac{N_{l}}{2}\left(\begin{array}{ll}
-X_{(l m)} & W_{(l m)} \sin \theta \\
W_{(l m)} \sin \theta & X_{(l m)} \sin ^{2} \theta
\end{array}\right),
\end{aligned}
$$

with the definitions

$$
\begin{aligned}
W_{(l m)} & \equiv\left(\frac{\partial^{2}}{\partial \theta^{2}}-\cot \theta \frac{\partial}{\partial \theta}+\frac{m^{2}}{\sin ^{2} \theta}\right) Y_{(l m)}, \\
X_{(l m)} & \equiv \frac{2 i m}{\sin \theta}\left(\frac{\partial}{\partial \theta}-\cot \theta\right) Y_{(l m)} .
\end{aligned}
$$

The exchange symmetry $\{Q, U\} \leftrightarrow\{U,-Q\}$ as $\mathrm{G} \leftrightarrow \mathrm{C}$ indicates that $Y_{(l m) a b}^{\mathrm{G}}$ and $Y_{(l m) a b}^{\mathrm{C}}$ represent polarizations rotated by $45^{\circ}$.

A most useful property of the $\mathrm{G} / \mathrm{C}$ decomposition is that, in linear theory, scalar perturbations can produce only G-type polarization and not C-type polarization. This is in contrast to tensor or vector metric perturbations which will produce a mixture of both types. To understand why scalar metric perturbations do not produce a C-type polarization pattern, consider a scalar perturbation with single Fourier mode $\mathbf{k}$ in the $\hat{\mathbf{z}}$ direction. The polarization in a given direction can be represented by a magnitude $\mathcal{P}=\left(Q^{2}+U^{2}\right)^{1 / 2}$ and an orientation angle $\alpha$ from the axis defining the Stokes parameters (here, choose $\hat{\theta}$ ), where $\tan 2 \alpha=U / Q$. For scalar perturbations, the orientation of the polarization can be determined only by the direction of $\mathbf{k}$ : thus $\alpha=0$ if the polarization orientation is along the direction of $\mathbf{k}$, or $\alpha=\pi / 2$ if the orientation is perpendicular to the direction of $\mathbf{k}$. In either case, in a given region of the sky all of the orientations are parallel and thus the polarization pattern has no curl. Since the curl is a linear operator, summing over Fourier modes does not alter this conclusion. For tensor and vector perturbations, the azimuthal symmetry in the scalar case is explicitly broken, and thus the Fourier vector does not completely define the direction of the polarization orientation. Another way to state this argument is that scalar perturbations have no handedness so they cannot produce any curl, whereas vector and tensor perturbations do have a handedness and therefore can.

Finding a nonzero component of C-type polarization in the $\mathrm{CMB}$ would provide compelling evidence for significant contribution of either vector or tensor perturbations at the time of last scattering. Given a polarization map of even a small part of the sky one could in principle test for vector or tensor contribution by computing the combination of derivatives of the polarization given by $\mathcal{P}_{: b c}^{a b} \epsilon_{a}^{c}$ which will be nonzero only for C-type polarization, i.e., when vector or tensor perturbations are present. Similarly only G-type polarization contributes to $\mathcal{P}^{a b} a b$. Of course, taking derivatives of noisy data is problematic; more robust measures are given below.

We now turn to statistics of CMB polarization. If the cosmological inhomogeneities are Gaussian random noise, then to the extent linear theory is valid, the CMB fluctuations will also be Gaussian random noise. Regardless of 
whether the distribution is Gaussian, rotational invariance requires that the two-point correlations be of the form

$$
\begin{aligned}
& \left\langle a_{(l m)}^{\mathrm{T} *} a_{\left(l^{\prime} m^{\prime}\right)}^{\mathrm{T}}\right\rangle=C_{l}^{\mathrm{T}} \delta_{l l^{\prime}} \delta_{m m^{\prime}}, \\
& \left\langle a_{(l m)}^{\mathrm{G} *} a_{\left(l^{\prime} m^{\prime}\right)}^{\mathrm{G}}\right\rangle=C_{l}^{\mathrm{G}} \delta_{l l^{\prime}} \delta_{m m^{\prime}}, \\
& \left\langle a_{(l m)}^{\mathrm{C} *} a_{\left(l^{\prime} m^{\prime}\right)}^{\mathrm{C}}\right\rangle=C_{l}^{\mathrm{C}} \delta_{l l^{\prime}} \delta_{m m^{\prime}}, \\
& \left\langle a_{(l m)}^{\mathrm{T} *} a_{\left(l^{\prime} m^{\prime}\right)}^{\mathrm{G}}\right\rangle=C_{l}^{\mathrm{TG}} \delta_{l l^{\prime}} \delta_{m m^{\prime}}, \\
& \left\langle a_{(l m)}^{\mathrm{T} *} a_{\left(l^{\prime} m^{\prime}\right)}^{\mathrm{C}}\right\rangle=C_{l}^{\mathrm{TC}} \delta_{l l^{\prime}} \delta_{m m^{\prime}}, \\
& \left\langle a_{(l m)}^{\mathrm{G} *} a_{\left(l^{\prime} m^{\prime}\right)}^{\mathrm{C}}\right\rangle=C_{l}^{\mathrm{GC}} \delta_{l l^{\prime}} \delta_{m m^{\prime}} .
\end{aligned}
$$

If we also require that the distribution of inhomogeneities be invariant under parity, then $C_{l}^{\mathrm{TC}}=C_{l}^{\mathrm{GC}}=0$ since the $Y_{(l m)}$ and the $Y_{(l m) a b}^{\mathrm{G}}$ have parity $(-1)^{l}$ while the $Y_{(l m) a b}^{\mathrm{C}}$ have parity $(-1)^{l+1}$. Measuring a nonzero $C_{l}^{\mathrm{TC}}$ and/or $C_{l}^{\mathrm{GC}}$ would be quite interesting, indicating a handedness to the inhomogeneities in our universe. However, we do not expect this and will henceforth only consider the four angular power spectra $\left\{C_{l}^{\mathrm{T}}, C_{l}^{\mathrm{G}}, C_{l}^{\mathrm{C}}, C_{l}^{\mathrm{TG}}\right\}$. The first is the well-known angular power spectrum of temperature anisotropies while the last three, new to this paper, are related to various quantities in previous work (see Ref. [10]). Note that the scalar, vector, and tensor contribution to each of the $C_{l}$ 's adds in quadrature, i.e., for $X=\mathrm{T}, \mathrm{G}, \mathrm{C}, \mathrm{TG}$

$$
C_{l}^{X}=C_{l}^{X \text { scalar }}+C_{l}^{X \text { vector }}+C_{l}^{X \text { tensor }},
$$

and this is true whether or not the fluctuations are Gaussian. We have argued that $C_{l}^{\mathrm{Cscalar}}=0$.

Given an all-sky temperature-polarization map, one can determine the $a_{(\mathrm{lm})}$ 's using Eq. (3), and then construct estimators for the $C_{l}$ 's in the usual way, i.e.,

$$
\begin{aligned}
\widehat{C_{l}^{\mathrm{T}}}=\sum_{m=-l}^{l} \frac{\left|a_{(l m)}^{\mathrm{T}}\right|^{2}}{2 l+1}, & \widehat{C_{l}^{\mathrm{G}}}=\sum_{m=-l}^{l} \frac{\left|a_{(l m)}^{\mathrm{G}}\right|^{2}}{2 l+1}, \\
\widehat{C_{l}^{\mathrm{C}}}=\sum_{m=-l}^{l} \frac{\left|a_{(l m)}^{\mathrm{C}}\right|^{2}}{2 l+1}, & \widehat{C_{l}^{\mathrm{TG}}}=\sum_{m=-l}^{l} \frac{a_{(l m)}^{\mathrm{T} *} a_{(l m)}^{\mathrm{G}}}{2 l+1} .
\end{aligned}
$$

If only part of the sky is mapped, the same techniques developed to analyze anisotropy with incomplete sky coverage [11] may be applied to polarization to construct other estimators of the various $C_{l}$ 's. The mean square polarization is

$$
\overline{Q^{2}+U^{2}}=2 \overline{\mathcal{P}^{a b} \mathcal{P}_{a b}}=\mathcal{P}_{\mathrm{G}}^{2}+\mathcal{P}_{\mathrm{C}}^{2},
$$

where

$$
\frac{\overline{\mathcal{P}_{\mathrm{G}}^{2}}}{T_{0}^{2}}=\sum_{l=2}^{\infty} \frac{2 l+1}{8 \pi} \widehat{C_{l}^{\mathrm{G}}}, \quad \frac{\overline{\mathcal{P}_{\mathrm{C}}^{2}}}{T_{0}^{2}}=\sum_{l=2}^{\infty} \frac{2 l+1}{8 \pi} \widehat{C_{l}^{\mathrm{C}}} .
$$

Since scalar modes do not contribute to $\overline{\mathcal{P}_{\mathrm{C}}^{2}}$, this statistic provides a powerful and unambiguous model-independent probe of tensor and vector perturbations.

To test a given spectrum of tensor modes against a polarization map, comparing the complete set of predicted $C_{l}^{\mathrm{C}}$ with the estimators $\widehat{C_{l}^{\mathrm{C}}}$ is more powerful than considering only $\mathcal{P}_{\mathrm{C}}^{2}$, if the detection has sufficient signal to noise. Usually, however, the theory being tested has scalar as well as nonscalar modes, along with undetermined cosmological parameters. If so, the most information can be extracted from the map by comparing the entire set of predicted moments, $\left\{C_{l}^{\mathrm{T}}, C_{l}^{\mathrm{G}}, C_{l}^{\mathrm{C}}, C_{l}^{\mathrm{TG}}\right\}$, with the measured estimators $[12,13]$.

Note that only the $C_{l}^{\mathrm{C}}$ 's potentially allow detection of a small vector or tensor signal. If scalar perturbations dominate, then the vector or tensor signal in $\left\{C_{l}^{\mathrm{T}}, C_{l}^{\mathrm{G}}, C_{l}^{\mathrm{TG}}\right\}$ may be swamped by the cosmic variance in the scalar signal, but the $C_{l}^{\mathrm{C}}$ 's are not contaminated in this way. The cross-correlation moments $C_{l}^{\mathrm{TG}}$, which differ for scalar, vector, and tensor perturbations [14], will be larger than the polarization autocorrelation moments. Therefore, the temperature-polarization cross correlation may be measured with some precision.

Much of the small-angle formalism of Refs. [3-5,1417] can be reproduced by replacing the $Y_{(I m)}(\hat{\mathbf{n}})$ 's in our formalism with Fourier modes, $e^{i \mathbf{l} \cdot \hat{\mathbf{n}}}$, and using regular derivatives rather than covariant ones. This small-angle formalism is completely analogous to that developed above and will provide an accurate description of a region of sky small enough to be approximated by a flat surface. The G/C decomposition in the small-angle formalism can be used to detect nonscalar perturbations on small scales, though the tensor and vector signal are liable to drop off rapidly at angular scales smaller than a few degrees.

To make contact with previous work, we can write the two-point temperature and polarization correlation functions [3-5,14-17] in terms of multipole moments [10]. Although correlation functions of Stokes parameters which appear in the previous literature depend on the positions of the points being correlated, rotationally invariant correlation functions exist which are closely related to those discussed above. To construct them, define Stokes parameters $Q_{r}$ and $U_{r}$ with respect to axes which are parallel and perpendicular to the great arc (or geodesic) which connects the two points being correlated. The two-point correlation functions are

$$
\begin{aligned}
C^{T}(\theta)= & T_{0}^{-2}\left\langle T\left(\hat{\mathbf{n}}_{1}\right) T\left(\hat{\mathbf{n}}_{2}\right)\right\rangle_{\hat{\mathbf{n}}_{1} \cdot \hat{\mathbf{n}}_{2}=\cos \theta} \\
= & \sum_{l} \frac{2 l+1}{4 \pi} C_{l}^{\mathrm{T}} P_{l}(\cos \theta), \\
C^{Q}(\theta)= & T_{0}^{-2}\left\langle Q_{r}\left(\hat{\mathbf{n}}_{1}\right) Q_{r}\left(\hat{\mathbf{n}}_{2}\right)\right\rangle_{\hat{\mathbf{n}}_{1} \cdot \hat{\mathbf{n}}_{2}=\cos \theta} \\
= & \sum_{l} \sqrt{\frac{2 l+1}{2 \pi}} N_{l}\left[C_{l}^{\mathrm{G}} W_{(l 2)}(\theta, 0)\right. \\
& \left.-i C_{l}^{\mathrm{C}} X_{(l 2)}(\theta, 0)\right], \\
C^{U}(\theta)= & T_{0}^{-2}\left\langle U_{r}\left(\hat{\mathbf{n}}_{1}\right) U_{r}\left(\hat{\mathbf{n}}_{2}\right)\right\rangle_{\hat{\mathbf{n}}_{1} \cdot \hat{\mathbf{n}}_{2}=\cos \theta} \\
= & \sum_{l} \sqrt{\frac{2 l+1}{2 \pi}} N_{l}\left[C_{l}^{\mathrm{C}} W_{(l 2)}(\theta, 0)\right. \\
& \left.-i C_{l}^{\mathrm{G}} X_{(l 2)}(\theta, 0)\right], \\
C^{T Q}(\theta)= & T_{0}^{-2}\left\langle T\left(\hat{\mathbf{n}}_{1}\right) Q_{r}\left(\hat{\mathbf{n}}_{2}\right)\right\rangle_{\hat{\mathbf{n}}_{1} \cdot \hat{\mathbf{n}}_{2}=\cos \theta} \\
= & \sum_{l} \frac{2 l+1}{4 \pi} N_{l} C_{l}^{\mathrm{TG}} P_{l}^{2}(\cos \theta),
\end{aligned}
$$


where $\theta$ is the angle separating the two points and $P_{l}^{2}$ is the $m=2$ associated Legendre function. Since $Q_{r}$ and $T$ are invariant under reflection along the great arc connecting the two points while $U_{r}$ changes sign, $\left\langle Q_{r} U_{r}\right\rangle=\left\langle U_{r} T\right\rangle=0$ if statistical invariance under parity holds. Equations (13) reduce to the correct small-angle formulas [17] when $\theta \ll$ 1. The functions $\left\{C^{T}(\theta), C^{Q}(\theta), C^{U}(\theta), C^{T Q}(\theta)\right\}$ are a different way of representing $\left\{C_{l}^{\mathrm{G}}, C_{l}^{\mathrm{T}}, C_{l}^{\mathrm{C}}, C_{l}^{\mathrm{TG}}\right\}$ and vice versa. In Gaussian models either set provides a complete statistical description of the temperature and polarization patterns.

The largest hurdles to detecting and characterizing CMB polarization are sensitivity and foregrounds. In adiabatic models with standard recombination the polarization is only a few percent of the anisotropy, although it may be larger in reionized [16], isocurvature, or topological-defect models. Thus the polarization signal is at least an order of magnitude below current experimental sensitivities. Experiments planned or envisioned over the coming decade, however, will likely attain the raw sensitivity necessary for detailed polarization investigations. Polarized emission from foreground sources is a relatively unknown factor at this time. Foreground emission and any Faraday rotation will certainly contribute to the C-type polarization, but these contaminants can be subtracted using multifrequency observations. On subdegree scales, where the signal from vector and tensor modes are liable to be negligible, any measurable C-type polarization is a likely indicator of contamination. Polarization measurements will be difficult, but the promise of using them to detect gravity waves or vorticity, and hence to discriminate between cosmological models, makes these measurements potentially very valuable.

During the preparation of this paper, we became aware of similar work by Seljak and Zaldarriaga [18]. We thank G. Jungman and U. Seljak for helpful discussions. This work was supported by D.O.E. Contract No. DEFG02-92ER 40699, NASA NAG5-3091, and the Alfred P. Sloan Foundation at Columbia, NASA AST94-19400 at FNAL, and the Harvard Society of Fellows. M.K. acknowledges the hospitality of the NASA/Fermilab Astrophysics Center and the CERN Theory Group. A.S. acknowledges the hospitality of the Aspen Center for Physics and the support of the NASA Grant No. NAG 5-2788.

*Electronic address: kamion@phys.columbia.edu †Electronic address: akosowsky@cfa.harvard.edu

\#Electronic address: stebbins@perseus.fnal.gov

[1] L. F. Abbott and M. Wise, Nucl. Phys. B244, 541 (1984).

[2] A. R. Liddle and D. Lyth, Phys. Lett. B 291, 391 (1992); R. L. Davis et al., Phys. Rev. Lett. 69, 1856 (1992).

[3] J. R. Bond and G. Efstathiou, Astrophys. J. Lett. 285, L47 (1984); Mon. Not. R. Astron. Soc. 226, 655 (1987); M. Zaldarriaga and D. Harari, Phys. Rev. D 52, 3276 (1995).

[4] A. G. Polnarev, Sov. Astron. 29, 607 (1985); R. Crittenden, R. L. Davis, and P. J. Steinhardt, Astrophys. J. Lett. 417, L13 (1993); D. Harari and M. Zaldarriaga, Phys. Lett. B 319, 96 (1993).

[5] A. Kosowsky, Ann. Phys. (N.Y.) 246, 49 (1996).

[6] A. Melchiorri and N. Vittorio, Report No. astro$\mathrm{ph} / 9610029$.

[7] See the MAP home page, URL http://map.gsfc.nasa.gov.

[8] F. J. Zerilli, J. Math. Phys. 7, 2203 (1970); K. S. Thorne, Rev. Mod. Phys. 52, 299 (1980).

[9] A. Stebbins, Report No. astro-ph/9609149 (to be published).

[10] M. Kamionkowski, A. Kosowsky, and A. Stebbins, Report No. astro-ph/9611125 (to be published).

[11] J. R. Bond, Phys. Rev. Lett. 74, 4369 (1995); N. Wright et al., Astrophys. J. Lett. 464, L21 (1996); M. Tegmark, Astrophys. J. Lett. 464, L35 (1996).

[12] M. Kamionkowski, D. N. Spergel, and N. Sugiyama, Astrophys. J. Lett. 426, L57 (1994).

[13] G. Jungman, M. Kamionkowski, A. Kosowsky, and D. N. Spergel, Phys. Rev. Lett. 76, 1007 (1996); Phys. Rev. D 54, 1332 (1996).

[14] R. G. Crittenden, D. Coulson, and N. G. Turok, Phys. Rev. D 52, 5402 (1995).

[15] D. Coulson, R. G. Crittenden, and N. G. Turok, Phys. Rev. Lett. 73, 2390 (1994); K. L. Ng and K.-W. Ng, Astrophys. J. 456, 413 (1996).

[16] K. L. Ng and K.-W. Ng, Phys. Rev. D 51, 364 (1995); Astrophys. J. 445, 521 (1995).

[17] U. Seljak, Report No. astro-ph/9608131.

[18] U. Seljak and M. Zaldarriaga, preceding Letter, Phys. Rev. Lett. 78, 2054 (1997). 